\title{
Preclinical evaluation of spatial frequency domain-enabled wide-field quantitative imaging for enhanced glioma resection
}

\author{
Mira Sibai \\ Carl Fisher \\ Israel Veilleux \\ Jonathan T. Elliott \\ Frederic Leblond \\ David W. Roberts \\ Brian C. Wilson
}




\title{
Preclinical evaluation of spatial frequency domain-enabled wide-field quantitative imaging for enhanced glioma resection
}

\author{
Mira Sibai, ${ }^{a, b}$ Carl Fisher, ${ }^{\text {a,b }}$ Israel Veilleux, ${ }^{b}$ Jonathan T. Elliott, ${ }^{c}$ Frederic Leblond, ${ }^{d}$ David W. Roberts, ${ }^{e}$ and \\ Brian C. Wilson ${ }^{a, b, *}$ \\ aUniversity of Toronto, Department of Medical Biophysics, Faculty of Medicine, Ontario, Canada \\ bUniversity Health Network, Princess Margaret Cancer Center, Ontario, Canada \\ 'Dartmouth College, Thayer School of Engineering, New Hampshire, United States \\ dÉcole Polytechnique De Montreal, Department of Engineering Physics, Québec, Canada \\ ${ }^{e}$ Dartmouth Hitchcock Medical Center, Department of Neurosurgery, New Hampshire, United States
}

\begin{abstract}
Aminolevelunic acid-induced protoporphyrin IX (PpIX) fluorescence-guided resection (FGR) enables maximum safe resection of glioma by providing real-time tumor contrast. However, the subjective visual assessment and the variable intrinsic optical attenuation of tissue limit this technique to reliably delineating only high-grade tumors that display strong fluorescence. We have previously shown, using a fiber-optic probe, that quantitative assessment using noninvasive point spectroscopic measurements of the absolute PpIX concentration in tissue further improves the accuracy of FGR, extending it to surgically curable low-grade glioma. More recently, we have shown that implementing spatial frequency domain imaging with a fluorescent-light transport model enables recovery of two-dimensional images of [PpIX], alleviating the need for time-consuming point sampling of the brain surface. We present first results of this technique modified for in vivo imaging on an RG2 rat brain tumor model. Despite the moderate errors in retrieving the absorption and reduced scattering coefficients in the subdiffusive regime of $14 \%$ and $19 \%$, respectively, the recovered [PpIX] maps agree within $10 \%$ of the point $[\mathrm{PpIX}]$ values measured by the fiber-optic probe, validating its potential as an extension or an alternative to point sampling during glioma resection. ๑ 2017 Society of Photo-Optical Instrumentation Engineers (SPIE) [DOI: 10 .1117/1.JBO.22.7.076007]
\end{abstract}

Keywords: spatial frequency domain imaging; quantitative fluorescence imaging; protoporphyrin IX; diffuse reflectance spectroscopy; glioma.

Paper 170053RR received Jan. 23, 2017; accepted for publication Jun. 21, 2017; published online Jul. 11, 2017.

\section{Introduction}

Neurosurgery is an integral component of treating patients with glioma, a highly malignant brain tumor that arises from the glial cells. If the tumor is operable, surgery is the first line of defense and is also often used upon recurrence. The objectives are to obtain tissues for accurate histopathologic classification and staging, to debulk the tumor, and to achieve complete or near complete resection of the tumor as can be done safely. Our main interest is to improve completeness of resection as, while patient outcome including survival greatly depends on this, the success rate remains low despite MRI guidance. ${ }^{1}$ In high-grade gliomas, resecting $>98 \%$ of the bulk tumor detected by MRI results in improved progression-free survival rates and prolonged mean overall survival, ${ }^{1}$ whereas low-grade gliomas are potentially curable if complete resection is achieved. ${ }^{2}$ However, gliomas are usually infiltrative, so delineating the tumor margins and visualizing any residual disease after tumor debulking are significant impediments in both cases. This difficulty is further exacerbated by the need to preserve normal brain tissue to retain maximum neurological function. As a result, there is a clinical need in neurosurgery for new highly sensitive and specific techniques for residual cancer detection.

*Address all correspondence to: Brian C. Wilson, E-mail: wilson@ uhnresearch ca
Intraoperative fluorescence-guided resection (FGR) has been developed to enhance tumor visualization. ${ }^{3}$ While applicable across many tumor sites, FGR has been most advanced in glioma surgery, where it has been used both at the beginning of surgery to define tumor/normal tissue boundaries and at the near end of surgery (after debulking) to identify residual tumor tissue in the resection bed as well as satellite tumors that have migrated beyond the resection bed. ${ }^{3}$ Clinically, FGR has resulted in a significant increase in complete resection rates as estimated by MRI contrast enhancement postsurgery. ${ }^{4}$

The most widely used fluorophore in FGR of glioma is protoporphyrin IX (PpIX), a precursor in the heme-biosynthesis pathway that preferentially accumulates in tumor cells upon administration of 5-aminolevelunic acid (ALA). ${ }^{5}$ There is generally excellent contrast between tumor and normal brain using ALA-PpIX fluorescence detection. ${ }^{5}$ However, to date, fluorescence visualization as implemented commercially in, e.g., the Zeiss Blue 400 neurosurgical microscope has been largely qualitative and subjective. This has limited resection of high-grade tumors to only the "strongly fluorescing" regions, likely leaving the more infiltrative components unresected. The qualitative nature of the detected signals has also led to inadequate sensitivity for reliable FGR of low-grade gliomas, which explains

$1083-3668 / 2017 / \$ 25.00$ (c) 2017 SPIE 
why PpIX FGR has been used clinically only for high-grade disease. $^{6}$

Significant challenges remain in making FGR a highly sensitive and quantitative procedure, including accounting for instrument-dependent distortions and calibrations and, more importantly, correcting the "raw" fluorescence signal for the marked effects of absorption and elastic scattering by the tissue of both the excitation (usually in the blue spectral range for PpIX, $\sim 400$ to $450 \mathrm{~nm}$ ) and emission light (usually $>\sim 630 \mathrm{~nm}$ for PpIX). As tissue optical properties are highly variable, both between patients and from point to point in each patient, it is necessary to measure these at the same time and location as the fluorescence measurements. ${ }^{6}$

We have developed several different platforms for quantitative PpIX FGR, i.e., to measure and map the absolute PpIX concentration, $[\mathrm{PpIX}]$, in tissue. ${ }^{7-9}$ There are two main approaches: semiempirical correction for tissue attenuation, typically using a form of multiple-wavelength ratiometry, and rigorous correction based on independent measurements of the tissue absorption and scattering coefficients at the relevant wavelengths. This study is an extension of two previous developments. The first was the use of hyperspectral fluorescence imaging together with semiempirical correction algorithms ${ }^{8,9}$ in which the use of a high-sensitivity electron-multiplying charge-coupled detector (EMCCD) enabled PpIX detection down to $10 \mathrm{ng} / \mathrm{ml}$ in a glioma model. ${ }^{9}$ The second development was based on an intraoperative quantitative point-contact fiber-optic spectroscopy probe $^{7}$ that has been shown in clinical trials to significantly improve the sensitivity and specificity of residual tumor tissue detection in high-grade glioma and to make FGR of a low-grade tumor feasible in patients. ${ }^{6}$ This intraoperative probe combines fluorescence spectroscopy with diffuse reflectance spectroscopy, where the latter is performed at two different sourcedetector separations on the tissue surface, allowing the absorption and transport scattering spectra to be calculated by applying a spectrally constrained light diffusion model. ${ }^{7}$ Using a spectral unmixing algorithm, the background tissue autofluorescence can be subtracted and, knowing the PpIX extinction spectrum, [PpIX] can be calculated with an accuracy of about $\pm 10 \%$ down to a concentration of $10 \mathrm{ng} / \mathrm{ml}$ by correcting the fluorescence signal using a model-based attenuation factor with the absorption and scattering coefficients as input parameters. This instrument is currently undergoing multicenter clinical trials. However, the point technique is clearly limited in practice by undersampling of tissue, while the use of hyperspectral imaging has unknown robustness given the inherent limitations of nonmodeled-based ratiometric techniques in terms of accuracy. The goal here was to combine the strengths of rigorous quantification with the speed of wide-field imaging. This requires mapping the tissue absorption and transport scattering coefficients, $\mu_{\mathrm{a}}$ and $\mu_{\mathrm{s}}^{\prime}$, respectively, and using these values to compute an attenuation correction factor for every imaged pixel.

Spatial frequency domain imaging (SFDI) is an emerging wide-field technique that has the capability to extract quantitative metrics relevant for fast and noninvasive diagnosis, such as biochemical and structural composition of tissue. ${ }^{10}$ SFDI can produce quantitative spatial maps of tissue absorption and scattering properties by projecting sinusoidal light patterns of different spatial frequencies, $f_{x}$, onto the tissue surface by detecting the spatially modulated diffuse reflectance images as a function of frequency. ${ }^{10}$ As tissue effectively acts as a low-pass spatial filter, the technique can be used to probe different tissue depths and optical properties. At lower spatial frequencies, corresponding to longer optical path lengths in the tissue, the modulation amplitude is sensitive to both absorption and multiple scattering, whereas at higher spatial frequencies, this amplitude is mostly sensitive to scattering within relatively smaller penetration depths. ${ }^{10}$ Detecting the diffuse reflectance at low and high spatial frequencies is analogous to measuring the diffuse reflectance at different source-detector separations using point detection techniques but executed in the spatial frequency domain. ${ }^{11}$ In the diffusion light transport regime, the spatially modulated diffuse reflectance is fitted to a light diffusion model in the spatial frequency domain to solve inversely for $\mu_{\mathrm{a}}$ and $\mu_{\mathrm{s}}^{\prime}$. A detailed description of SFDI to recover tissue optical properties is presented in Ref. 10. In the operating room, the utility of SFDI as a quantitative tissue analyzer has been applied in reconstructive surgery, where the metric of interest is real-time measurement of the hemoglobin oxygenation status and content to assess tissue viability. ${ }^{12,13}$ More recently, the measured tissue scattering has been used to better identify tumor margins during breast conservative surgery, based on structural differences between tumor and normal tissue. ${ }^{13}$

A potential application of SFDI is leveraging its capabilities toward achieving quantitative, wide-field FGR. An ex vivo study by Yang et al. ${ }^{14}$ used SFDI to correct the measured fluorescence images for the effects of tissue absorption, by normalizing the spatially modulated fluorescence images by the corresponding diffuse reflectance images at a relatively high spatial frequency of $0.5 \mathrm{~mm}^{-1}$. Perhaps more relevant to the development of FGR, a preclinical glioma study by Konecky et al. ${ }^{15}$ exploited the secondary absorption peak of PpIX at $635 \mathrm{~nm}$ to excite tissue with red-light patterns (enabling greater penetration in tissue) and applied a tomographic approach to decouple [PpIX] from the tumor depth. A significant limitation is that an approximate measure of depth needs to be known a priori. ${ }^{15}$ To our knowledge, no other in vivo study has reported the use of SFDI for quantitative PpIX fluorescence imaging under the conditions of FGR as it is currently practiced in neurosurgery, i.e., subdiffusive imaging under violet/blue light excitation. Other studies, such as Saager et al., ${ }^{16}$ utilized near-infrared excitation and fluorescence detection in the fully diffusive regime, where autofluorescence and PpIX photoproducts were not spectrally separated from the intrinsic PpIX fluorescence signal, thereby producing only relative estimates of [PpIX]. One potential issue for PpIX FGR is that the optical properties of malignant brain tissue in the violet/blue wavelength range lie in the subdiffusive regime, ${ }^{6}$ where $\mu_{\mathrm{a}} \sim \mu_{\mathrm{s}}^{\prime}$, render the reduced transport albedo, $a^{\prime}=\frac{\mu_{\mathrm{s}}^{\prime}}{\left(\mu_{\mathrm{a}}+\mu_{\mathrm{s}}^{\prime}\right)} \ll 1$, so standard diffusion theory may not be sufficiently accurate. This definition of subdiffusive regime is not to be confused with subdiffuse scattering, where photons are detected after only a single or few scattering events occur. In that case, scattering parameters characterizing ultraand microbiostructures are of interest (see, for example, Kanick et al. ${ }^{17}$ ).

We present our first results of implementing spatial frequency domain-enabled quantitative fluorescence imaging (SFD-qFI) in an intracranial glioma model in rats, extending our previous work in tissue-simulating phantoms. ${ }^{18}$ In that work, we demonstrated the ability to recover $\mu_{\mathrm{a}}$ and $\mu_{\mathrm{s}}^{\prime}$ in the subdiffusive regime with accuracies of $\pm 8 \%$ and $\pm 13 \%$, respectively. ${ }^{18}$ Here, a model-based SFDI algorithm is used for two-dimensional (2-D) mapping of the wavelength-dependent tissue optical properties, $\mu_{\mathrm{a}}$ and $\mu_{\mathrm{s}}^{\prime}$, in the brain/tumor, 
and these are then used as inputs to a fluorescent-light transport model to convert the raw fluorescence images to absolute [PpIX] images. We show that, while the diffusion-based SFDI model has limited accuracy in deriving the tissue optical properties in the subdiffusive regime, we can nevertheless map [PpIX] with similar sensitivity and accuracy to our gold-standard point-probe system.

\section{Materials and Methods}

\subsection{Quantitative Point Fluorescence Spectroscopy}

The right side of Fig. 1(b) shows the intraoperative probe affixed to a vertical 3-D translation stage. This instrument has been described in detail previously. ${ }^{19}$ Briefly, the probe consists of four $200-\mu \mathrm{m}$ core-diameter optical fibers spaced $260-\mu \mathrm{m}$ apart center to center; two fibers are attached to broadband white light sources; one is attached to a blue/violet light-emitting diode (LED) centered at $405 \mathrm{~nm}$, and the other is a collection fiber connected to a spectrometer. ${ }^{19}$

The measured diffuse reflectance for each source-detector separation is fitted to the spectrally constrained diffuse reflectance predicted by diffusion theory. The spectral shapes of $\mu_{\mathrm{a}}$ and $\mu_{\mathrm{s}}^{\prime}$ are assumed to be known a priori for a given tissue type and serve as fitting constraints: hemoglobin is assumed to be the dominant absorber, while the transport scattering follows an inverse power law as a function of wavelength. For excitation at 400 to $440 \mathrm{~nm}$, where PpIX and hemoglobin absorption are high, the remitted fluorescent photons, $F_{m}$, detected at 600 to $720 \mathrm{~nm}$ can be assumed to travel similar path lengths to those for the diffuse reflectance signal, $R_{m}$, in the same wavelength range. Hence, $F_{m}$ is proportional to $R_{m}$ multiplied by the fraction of excitation photons that are absorbed by PpIX and that produce fluorescence. Knowing the main endogenous fluorophores in brain tissue [(nicotinamide adenine dinucleotide (NADH), lipofuscin, and flavin adenin dinucleotide (FAD)] the total quantitative fluorescence signal is then spectrally unmixed to separate PpIX fluorescence from autofluorescence as well as from any PpIX photoproducts generated during surgical exposure of the tissue. Given the known PpIX extinction spectrum, the corrected PpIX spectrum can then be used to determine [PpIX]. In this work, we apply the algorithm developed for point measurements to achieve SFD-qFI.

\subsection{Quantitative Reflectance Imaging}

\subsubsection{SFDI and experimental setup}

A detailed description of the SFD-qFI system has been presented previously. ${ }^{18}$ Briefly, illumination comprises six LEDs (Spectra X: Lumencor, Beaverton, Oregon) centered at 390, $440,475,512,586$, and $632 \mathrm{~nm}$ and coupled to a 3-mm-diameter liquid light guide (LGG0338, ThorLabs, Montreal, Canada) to project the light onto a spatial light modulator (Digital Micromirror Device, 0.55XGA Series 450 DMD, Texas Instruments, Dallas, Texas) from which it is reflected onto the sample. The imaging system consists of a 14-bit chargecoupled device (CCD; Pixelfly, PCO AG, Kelheim, Germany) connected to a visible-range liquid crystal tunable filter (LCTF, varispec-07-02: Perkin Elmer, Waltham, Massachusetts) by a $2: 1$ relay C-mount lens. This optimally fills the CCD field of view across the rat brain $(\sim 2 \mathrm{~cm} \times 2.5 \mathrm{~cm})$. Crossed polarizers are included in both the projection and imaging paths to reduce specular reflection. Eight spatial frequencies, ranging from 0 to $1.0 \mathrm{~mm}^{-1}\left(0,0.05,0.1,0.2,0.3,0.4,0.5\right.$, and $\left.1 \mathrm{~mm}^{-1}\right)$ are projected at three spatial phases, $0,2 \pi / 3$, and $4 \pi / 3$ to recover the demodulated diffuse reflectance images. It takes $\sim 12 \mathrm{~s}$ to collect one set of images per phase shift for 32 wavelengths from 400 to $720 \mathrm{~nm}$ in $10-\mathrm{nm}$ steps. Figure 1 shows a schematic diagram and photograph of the SFD-qFI system with a rat affixed to the stereotaxic animal holder. Adjacent to the imaging system is the 3-D translational stage used to sample the brain surface with the contact probe to serve as the reference standard for local $[\mathrm{PpIX}]$ measurements.

\subsubsection{SFDI implementation and recovery of $\mu_{\mathrm{a}}$ and $\mu_{\mathrm{s}}^{\prime}$}

The structured light illumination patterns projected on the brain surface are sinusoids of the form

$I_{i}(x, y)=I_{\mathrm{DC}}+A \times \cos \left(2 \pi f_{x} \times x+\varphi_{i}\right)$,

with phase offsets of $\phi_{i}=\left[0, \frac{2 \pi}{3}, \frac{4 \pi}{3}\right]$ for $i=1,2,3 . f_{x}$ is the spatial frequency in $\mathrm{mm}^{-1}, I_{\mathrm{DC}}$ is the direct current (DC) component of the excitation light, and $A$ is the modulation depth. ${ }^{10}$ For each pixel, the modulated alternating current (AC) reflectance, $M_{\mathrm{AC}}$, is calculated from the set of intensity images as

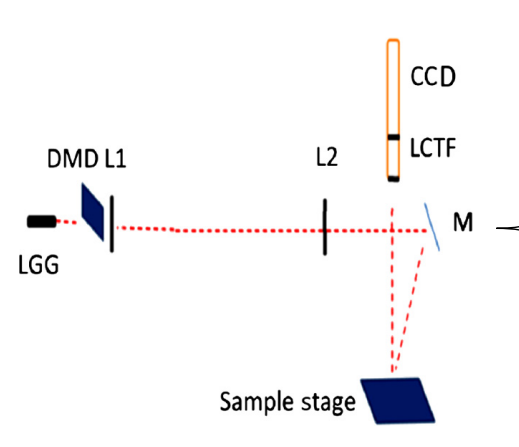

(a)

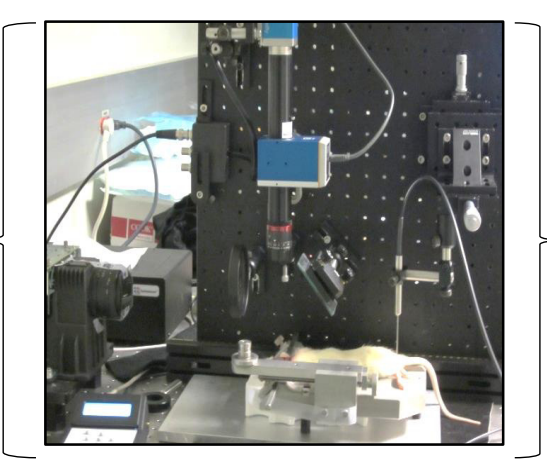

(b)
Intraoperative probe on a 3-D translational stage

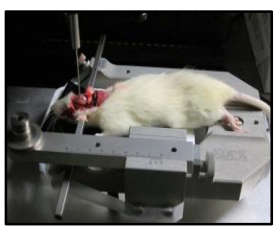

(c)

Fig. 1 SFD-qFI setup: (a) schematic diagram showing the liquid light guide, DMD, lenses (L1, L2), mirror (M), LCTF, CCD, and the sample stage, (b) photograph of the system as well as the point probe mounted on a 3-D translation stage, and (c) close-up of a rat affixed to the stereotaxic frame with the probe positioned for point qFS measurements. 
$M_{\mathrm{AC}}\left(x_{i}, f_{x}\right)=\sqrt{\frac{2 \times\left\{\left[I_{1}(x)-I_{2}(x)\right]^{2}+\left[I_{1}(x)-I_{3}(x)\right]^{2}+\left[I_{2}(x)-I_{3}(x)\right]^{2}\right\}}{3}}$.

The modulated DC reflectance amplitude, $M_{\mathrm{DC}}$, is obtained from the average of the three phase-shifted intensity images for the same $f_{x}$,

$M_{\mathrm{DC}}\left(x_{i}\right)=\frac{I_{1}\left(x_{i}\right)+I_{2}\left(x_{i}\right)+I_{3}\left(x_{i}\right)}{3}$.

The calibrated demodulated reflectance, $R_{\mathrm{d}}^{\text {sample }}$, is determined from the ratio of the demodulated AC intensity measured on the sample, $M_{\mathrm{AC}}^{\text {sample }}$, to the demodulation image obtained by projecting the same spatial frequencies onto a reference standard of known optical properties, $M_{\mathrm{AC}}^{\mathrm{ref}}$, multiplied by the diffuse reflectance, $R_{\mathrm{d}}^{\text {ref }}$, of that standard, as predicted by diffusion theory or determined by Monte Carlo simulations. Thus,

$R_{\mathrm{d}}^{\mathrm{sample}}\left(x_{i}, f_{x}\right)=\frac{M_{\mathrm{AC}}^{\mathrm{sample}}\left(x_{i}, f_{x}\right)}{M_{\mathrm{AC}}^{\mathrm{ref}}\left(x_{i}, f_{x}\right)} \times R_{\mathrm{d}}^{\mathrm{ref}}\left(x_{i}, f_{x}\right)$.

Using Eq. (4), the modulation response of the imaging system itself is decoupled from the tissue response, so $\mu_{\mathrm{a}}$ and $\mu_{\mathrm{s}}^{\prime}$ can then be extracted by inversely to solve the spatially resolved diffusion equation in the SFD. ${ }^{10}$

\subsection{Quantitative Fluorescence Imaging}

The blue LED centered at $440 \mathrm{~nm}$ is used to induce PpIX fluorescence. Hyperspectral fluorescence images are acquired for $300 \mathrm{~ms}$ per wavelength from 600 to $720 \mathrm{~nm}$ in increments of $10 \mathrm{~nm}$. Planar diffuse reflectance images at the excitation and emission wavelengths, $R_{x}$ (at $440 \mathrm{~nm}$ ) and $R_{m}$ (at $640 \mathrm{~nm}$ ), as well as 2-D maps of $\mu_{\mathrm{a}, x}$ and $\mu_{\mathrm{S}, \mathrm{X}}^{\prime}$ are recovered by SFDI [Eqs. (1)-(4)]. Those values are then used to retrieve the quantitative fluorescence $q f$ for each pixel using the formula ${ }^{7}$

$q f_{x, m}(x, y, \lambda)=\frac{\mu_{\mathrm{a}, x}(x, y) \times F_{m}(x, y, \lambda)}{\left[1-R_{x}(x, y)\right] \times R_{m}(x, y, \lambda)}$.

$[\mathrm{PpIX}]$ is extracted on a pixel-by-pixel basis in the same manner as for the point spectroscopy probe, yielding 2-D maps of $[\mathrm{PpIX}]$ that can be overlaid on the diffuse reflectance images.

\subsection{Postmortem and In Vivo Imaging}

\subsubsection{Tumor induction}

Female Fischer 344 rats (Envigo, Maryland) were used under institutional approval (University Health Network, Toronto, Canada). For tumor induction, the rats were anesthetized using a nose cone with $4 \%$ isoflurane in oxygen at $2 \mathrm{~L} / \mathrm{min}$ and maintained by $1 \%$ to $2.5 \%$ isoflurane at $1 \mathrm{~L} / \mathrm{min}$. The eyes were lubricated with tear gel and the animals were placed on a warming pad. The scalp was shaved and disinfected with betadine and isopropanol, followed by a $1.5-\mathrm{cm}$ incision along the midline to retract the scalp. A 1-mm burr hole was made in the left cranium, $3 \mathrm{~mm}$ posterior to the bregma and $3 \mathrm{~mm}$ to the left of the sagittal suture, exposing the dura but leaving it intact. Subsurface intracranial brain tumors were induced by injecting $10^{5} \mathrm{RG} 2$ (ATCC CRL-2433) cells in $5 \mu \mathrm{L}$ in Neurobasal-A media (Life Technologies, Massachusetts) through the burr hole to a depth of either $0.5 \mathrm{~mm}$ or 1.5 to $2 \mathrm{~mm}$ below the dura using a $30 \mathrm{G}$ Hamilton Neuros syringe. The burr hole was then closed with bone wax. After surgery, the animals were monitored continuously until full recovery, and buprenorphine $(0.05 \mathrm{mg} / \mathrm{kg} \mathrm{SQ})$ was administered for analgesia every $12 \mathrm{~h}$ for the first $72 \mathrm{~h}$. Tumors were allowed to grow for 13 to 16 days to 2 - to $4-\mathrm{mm}$ diameter.

\subsubsection{In situ and in vivo imaging}

In vivo imaging after removing the dura proved to be suboptimal, as this caused excessive bleeding that had to be continuously cleared with saline. Hence, craniotomy followed by SFD-qFI was performed immediately after euthanasia for the first set of rats $(N=6)$ for which cancer cells were injected 1.5- to 2-mm deep. This will henceforth be referred to below as in situ imaging. The craniotomy and removal of the dura were necessary in these animals to visualize the bulk tumor that tended to grow more axially (downward) than laterally. A second set of rats $(N=2)$ was used to test the feasibility of actual in vivo imaging. In this case, cancer cells were injected superficially ( 0.5-mm deep), allowing in vivo imaging following removal of the bone flap but not of the dura, thereby avoiding excessive bleeding.

On the day of surgery, a 50 or $100 \mathrm{mg} / \mathrm{kg}$ body weight (BW) dose of ALA (Sigma-Aldrich, Ontario, Canada) was injected intraperitoneally and the rats were then kept under subdued light for $1 \mathrm{~h}$. For in situ imaging, the animals were sacrificed using $120 \mathrm{mg} / \mathrm{kg} \mathrm{BW}$ of euthanyl (sodium pentobarbital $240 \mathrm{mg} / \mathrm{mL}$ ) via the tail vein under general anesthesia. The skull and dura were rapidly removed to expose the tumor. Each rat was immediately affixed to the stereotaxic animal frame and positioned for imaging [Fig. 1(b)]. Fiducial markers were placed in a lateral line across the brain [Fig. 2(c)] to guide the point qFS measurements in steps of $0.4 \mathrm{~mm}$, sampling the tumor as well as adjacent region and the contralateral normal brain. Following qFS, planar hyperspectral fluorescence images were acquired under blue light excitation. White light SFDI was also performed using eight spatial frequencies up to $1 \mathrm{~mm}^{-1}$ and SFD-qFI analysis was performed on three regions of interest (ROI) spanning the qFS point measurements: tumor, adjacent, and contralateral brain.

For the in vivo imaging, craniotomy was performed at $1 \mathrm{~h}$ after ALA injection, under the same general anesthesia as used for cancer cell injection. The scalp was shaved and betadine was applied. The bone flap was carefully removed keeping the dura intact. A pulse oximeter was used to monitor heart rate and oxygen saturation. The same steps followed for postmortem imaging were then followed. Multiple probe measurements were taken over both the tumor and the contralateral side. Post SFDqFI, the rats were sacrificed by $120 \mathrm{mg} / \mathrm{kg}$ euthanyl injection via the tail vein.

\subsubsection{Ex vivo imaging}

After letting the tumors grow for 13 to 16 days, $100 \mathrm{mg} / \mathrm{kg}$ ALA was injected $1 \mathrm{~h}$ prior to sacrifice as described above. 

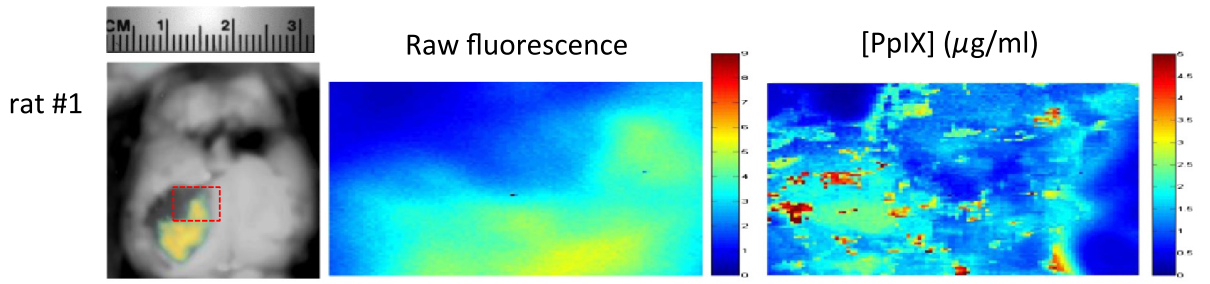

rat \#2
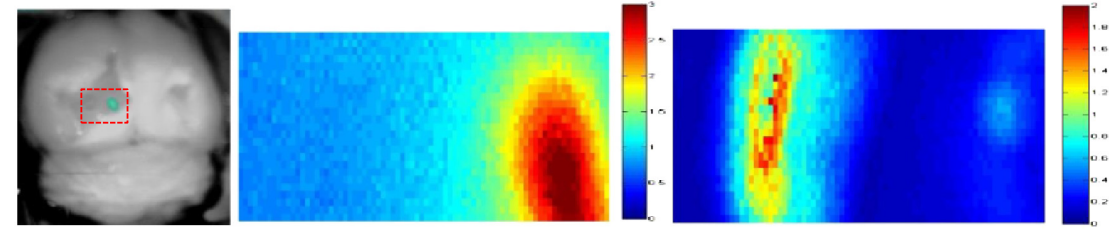

rat \#3

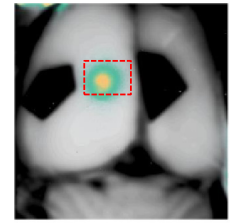

(a)

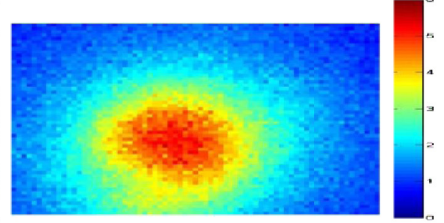

(b)

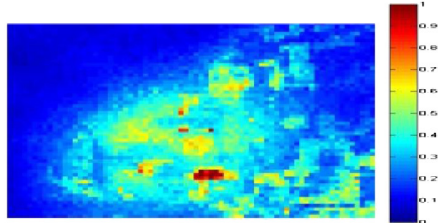

(c)

Fig. 2 In situ imaging: (a) raw fluorescence images overlaid on diffuse reflectance images, showing different tumor sizes and levels of infiltration, (b) and (c) show, respectively, magnified raw fluorescence images at $640 \mathrm{~nm}$ and derived [PpIX] images within tumor ROI indicated by the red dashed zones in (a). The intensity values on the color bars for raw fluorescence varied from 0 to 9,0 to 3 , and 0 to 6 for rats \#1 to 3 , respectively. [PpIX] values on the color bars for the third column varied from 0 to $5 \mu \mathrm{g} / \mathrm{ml}, 0$ to $2 \mu \mathrm{g} / \mathrm{ml}$, and 0 to $1 \mu \mathrm{g} / \mathrm{ml}$ for rats \#1 to 3 , respectively.

The whole brain $(N=4)$ was removed intact and 2 to $3-\mathrm{mm}$ thick coronal sections were cut at the tumor boundaries. The same imaging sequence used for in vivo imaging was followed, with the tissue section sampled first by qFS and then by SFD-qFI.

\section{Results}

Figure 2 shows examples of the raw in situ fluorescence images at $635 \mathrm{~nm}$ overlaid on the diffuse reflectance images at the same emission band under white light illumination as well as the corresponding $[\mathrm{PpIX}]$ map for the tumor regions. In the first two rats that had 2-mm depth of tumor cell injection, a thin layer of overlying normal brain was first resected to expose the tumor surface; this was not required in the remaining rats where the cells were injected only $1.5 \mathrm{~mm}$ below the dura so that the tumor grew up to the superficial layers of the brain surface. Although the rats were matched as much as possible in weight and age, there were marked variations in tumor size and degree of infiltration: e.g., rat \#2 had two tumor foci in which one showed no visible fluorescence but the corresponding [PpIX] image showed comparable concentration as the tumor that was visible under qualitative fluorescence imaging. Fluorimetry measurements in ex vivo tissue samples from both right and left foci confirmed the presence of tumor and significant [PpIX], corresponding to the SFD-qFI results. ${ }^{20}$ SFDI analysis showed that $\mu_{\mathrm{a}}$ was five times higher in the nonvisualized tumor (2.8 versus $0.56 \mathrm{~mm}^{-1}$ at $450 \mathrm{~nm}$ ) due to greater hemoglobin content. This demonstrates clearly the value of the qFS approach in accounting for variable tissue optical properties, as we have observed also in patients using the point probe. ${ }^{6}$ On the other hand, the quantitative [PpIX] map for rat \#1 showed a more heterogeneous PpIX accumulation across the tumor compared with the raw fluorescence image [Fig. 2(b)]. Additionally, the
[PpIX] image showed higher tumor-to-normal contrast, particularly at the tumor boundary, compared with the more diffuse boundary apparent in the raw fluorescence image. In rat \#3, the center of the tumor exhibited a raw fluorescence intensity that was 2.5 times higher than at the boundary, while the [PpIX] map showed a more uniform distribution (variation of only 1.5 -fold), while still depicting local heterogeneity throughout the tumor.

The local $[\mathrm{PpIX}]$ variations in rat \#3 are further shown in Fig. 3(b), where profiles of $\mu_{\mathrm{a}}, \mu_{\mathrm{s}}^{\prime}$, and [PpIX] are plotted along the line of the point-probe measurements from left to right [dashed line in Fig. 3(a)]. All three profiles show good correspondence between the SFDI and point-probe measurements and illustrate also the heterogeneity of the calculated [PpIX] maps compared with the raw fluorescence images, with significant PpIX present in the tissue adjacent to the tumor core, indicating tumor infiltration.

Ex vivo measurements were performed in intact resected brain (rats \#4 and 5) to validate the coregistration between qFI and qFS measurements. The tumor slices shown in Fig. 4(a) were oriented so that the top surface of the brain was positioned at the bottom of the image. The white light image shows a relatively dark and homogeneous tumor with a major blood vessel in the middle (estimated to be $0.4 \mathrm{~mm}$ below the surface by counting the number of pixels between the tumor boundary and the bottom edge of the tissue). The raw fluorescence image shows a step-like variation in the PpIX fluorescence, in marked contrast with the actual $[\mathrm{PpIX}]$ maps, where the higher $[\mathrm{PpIX}]$ at the middle of the tumor is correlated with higher tissue absorption coefficient $\left[5\right.$ versus $1 \mathrm{~mm}^{-1}$ : Figs. 4(d)-4(f)]. This is consistent with greater PpIX accumulation in regions with higher microvascular density. The bright granularity seen in (d) and (e) is in part an artifact due to light 


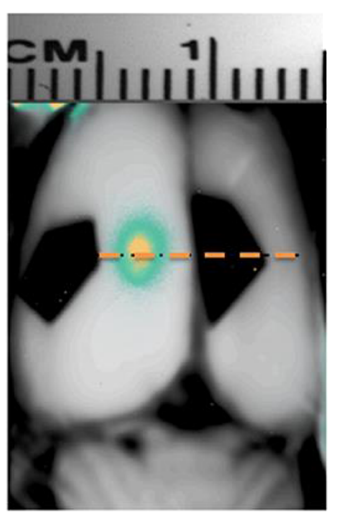

(a)
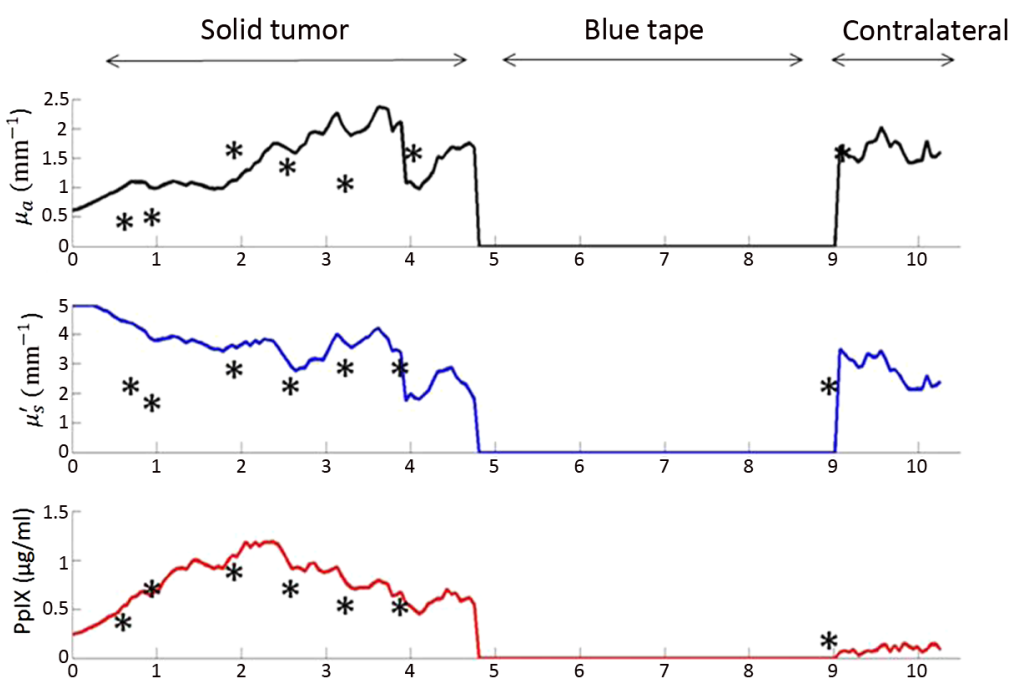

Distance starting from the tape in the left hemisphere to the edge of the right hemisphere $(\mathrm{mm})$

(b)

Fig. 3 Comparison of SFDI and qFS profiles: (a) raw fluorescence overlaid on the diffuse reflectance image for rat \#3 showing the tumor with hyperintense core (yellow) and surrounding diffuse signal at the tumor boundaries (green). The two black regions are pieces of surgical tape used to guide the point-probe translation, and (b) profiles (solid lines) of SFDI-derived $\mu_{\mathrm{a}}$ and $\mu_{\mathrm{S}}^{\prime}$ at $450 \mathrm{~nm}$ and [PpIX] compared with the qFS measurements indicated by *, also calculated at $450 \mathrm{~nm}$. Note that the tissue optical properties and $[\mathrm{PpIX}]$ values are absolute and have not been normalized between the two groups of measurements.

Coronal section with tumor included

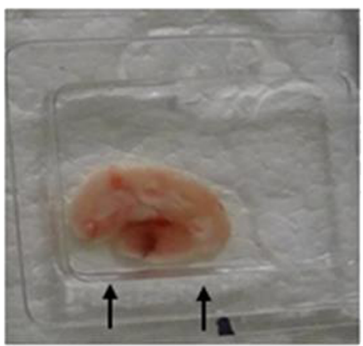

(a)
Reflectance at $620 \mathrm{~nm}$

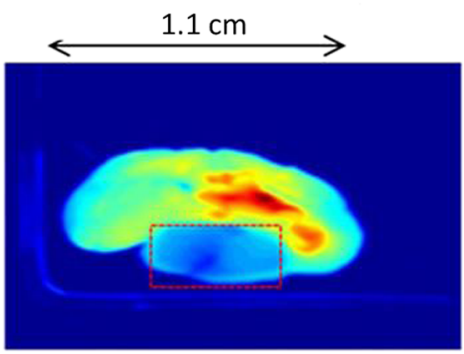

(b)

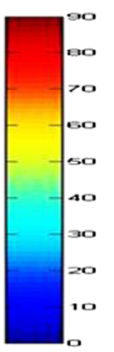

$\mu_{\mathrm{a}}$ at $450 \mathrm{~nm}\left(\mathrm{~mm}^{-1}\right)$

Fluorescence at $635 \mathrm{~nm}$ of tumor region

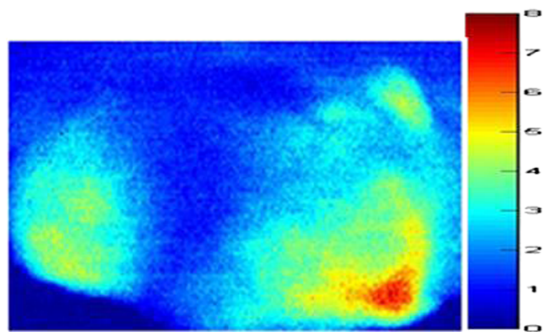

(c)

[PpIX] profile from $q F S$ and SFD-qFI

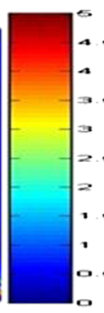

(d)

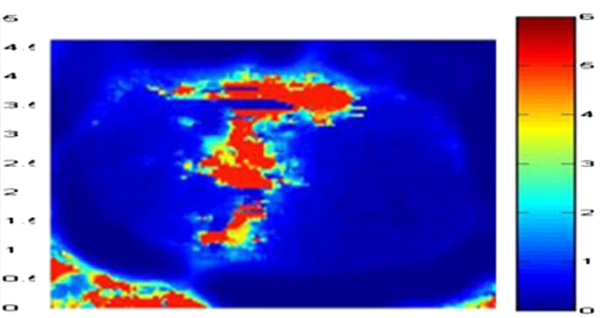

(e)

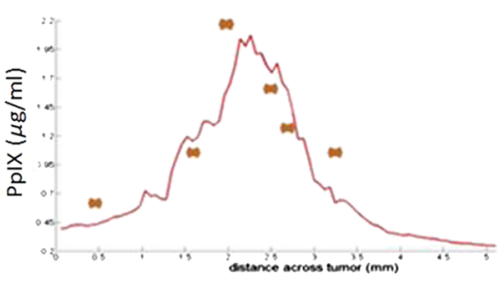

Distance from left to right $(\mathrm{mm})$

(f)

Fig. 4 Example of ex vivo fluorescence and reflectance images (rat \#4): (a) white light image of 3-mm thick coronal section: the tumor is located to the left of the black mark (tumor extent is depicted by two arrows) in this frame, (b) raw diffuse reflectance imaged at $620 \mathrm{~nm}$ with the tumor ROI indicated by the dashed box. Color bar intensity values varied from 0 to 90 (au), (c) raw fluorescence image (440-nm excitation and 635-nm emission) of the ROI shown in (b). Color bar intensity values varied from 0 to 8 (au), (d) corresponding SFD-qFI-derived image of [PpIX], which varied from 0 to $5 \mu \mathrm{g} / \mathrm{ml}$, (e) quantitative $\mu_{\mathrm{a}}$ map at $450 \mathrm{~nm}$ in the ROI obtained from SFDI, where values varied from 0 to $6 \mathrm{~mm}^{-1}$, and (f) corresponding [PpIX] profile calculated as the mean value of each column in (d) from SFD-qFI (solid line) and probe measurements (points). 
Diffuse reflectance

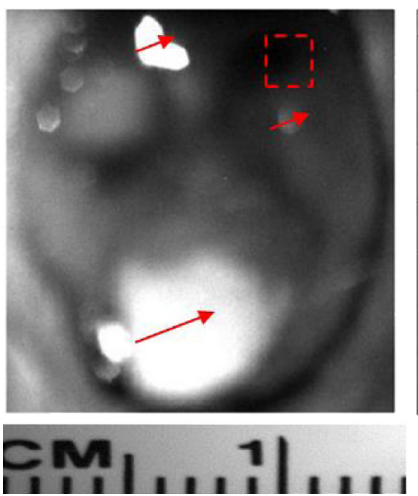

(a)
Fluorescence (au)

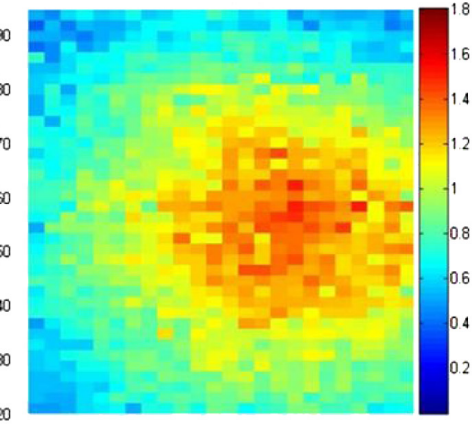

(b)

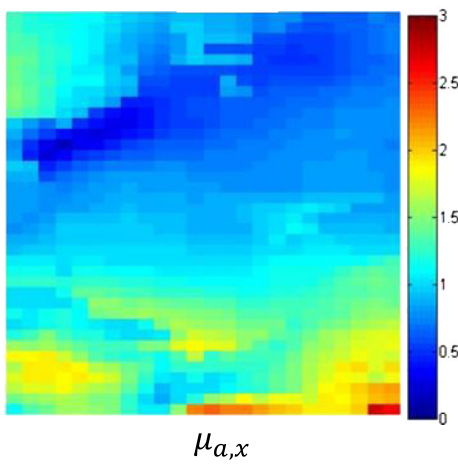

(d)

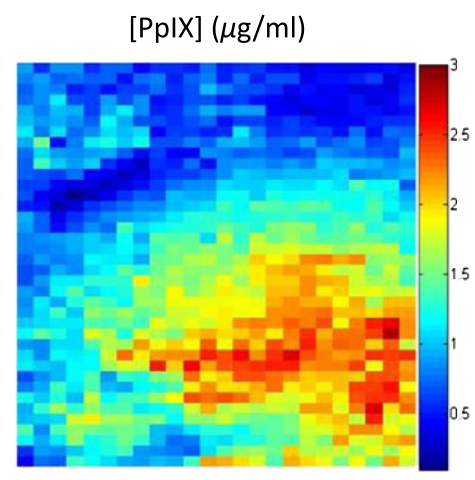

(c)

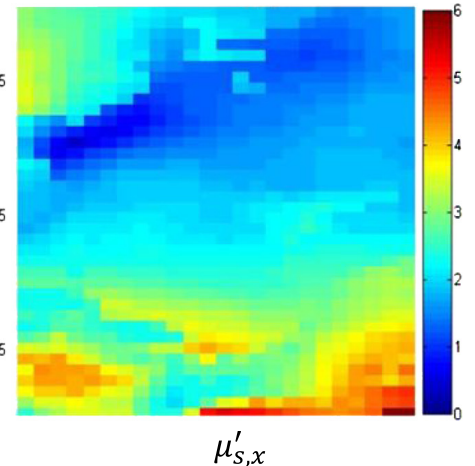

(e)

Fig. 5 In vivo quantitative imaging: (a) diffuse reflectance at $620 \mathrm{~nm}$ with the dura intact and the superficial tumor located within ROI indicated by the dashed box. The saturated regions are due to specular reflection (indicated by arrows), (b) raw fluorescence image at $635 \mathrm{~nm}$ in the ROI, (c) corresponding [PpIX] map, (d) $\mu_{\mathrm{a}}$ map at $450 \mathrm{~nm}$, and (e) $\mu_{\mathrm{s}}^{\prime}$ map at $450 \mathrm{~nm}$.

absorption by black flocked self-adhesive paper (TB4, Thorlabs, Montreal, Canada) used to minimize back scattering.

Figure 5 shows an example of in vivo imaging in a rat \#6 bearing a superficial intracranial tumor of 2.2-mm diameter as determined by preoperative MRI. The raw fluorescence at $635 \mathrm{~nm}$ could not be overlaid onto the diffuse reflectance image in Fig. 5(a) due to the specular reflection from the dura despite using crossed polarizers. However, the region around the tumor did not have any nearby specular reflection-induced pixel saturation. The mean absorption and reduced scattering coefficients of the tumor at $450 \mathrm{~nm}$ were $1.26 \pm 0.09 \mathrm{~mm}^{-1}$ and $2.98 \pm 0.14 \mathrm{~mm}^{-1}$, respectively, whereas the mean $[\mathrm{PpIX}]$ in the tumor was $1.45 \pm 0.25 \mu \mathrm{g} / \mathrm{ml}$. The corresponding values from the point-probe measurements were $1.28 \pm 0.38 \mathrm{~mm}^{-1}$, $2.26 \pm 0.72 \mathrm{~mm}^{-1}$, and $1.25 \pm 0.56 \mu \mathrm{g} / \mathrm{ml}$.

In Fig. 6, the mean $\mu_{\mathrm{a}}, \mu_{\mathrm{s}}^{\prime}$, and [PpIX] across the ROI for each rat are plotted against the values determined by the point probe, with the error bars indicating the standard deviation of each parameter within the tumor ROI for each rat. $\mu_{\mathrm{a}}$ and $\mu_{\mathrm{s}}^{\prime}$ at $405 \mathrm{~nm}$, determined by qFS and applied to calculate [PpIX], were then recalculated at $450 \mathrm{~nm}$ to make the comparison with those obtained by SFDI at the same wavelength more accurate (Fig. 6). The error bars for $\mathrm{qFS}$ are generally larger than for SFDI, likely because of the spatial heterogeneity of the PpIX fluorescence and the relative undersampling of the point probe (typically 10 locations) compared with the wide-field imaging, where each standard deviation was derived from 50 to
100 pixel values. The large error bars in $\mu_{\mathrm{a}}$ and $[\mathrm{PpIX}]$ for rat \#4 for both SFD-qFI and qFS are due to the high blood content in part of the tumor, similar to the ex vivo example shown in Fig. 4, whereas the measurements for rat \#6 were taken in vivo. There was no systematic trend between the absorption coefficient values measured by each technique, but the SFDI method appeared to somewhat overestimate the tissue scattering. This is probably due to the different source-detector separations used in qFS and SFDI, the former being the specific separations of 0.26 and $0.52 \mathrm{~mm}$ and the latter being dependent on the projected spatial frequency, which correspond to radial separations of 0.15 up to $3.15 \mathrm{~mm}$. Specifically, the smaller source-detector separation of $0.15 \mathrm{~mm}$ at the higher spatial frequencies used in SFDI will be more sensitive to detecting higher scattering events.

The mean relative differences between measuring $\mu_{\mathrm{a}}$ and $\mu_{\mathrm{s}}^{\prime}$ by SFDI and qFS were $14 \%$ and $19.4 \%$, respectively, probably due to the larger tissue volumes interrogated by SFDI at $440 \mathrm{~nm}$ compared with the point probe. The mean penetration depth of 405-nm excitation light in brain tissue is $\sim 0.25 \mathrm{~mm}$, whereas the mean penetration depth at $440-\mathrm{nm}$ excitation light is $\sim 0.45 \mathrm{~mm}$ as estimated by Monte Carlo. However, these differences did not propagate fully into the calculated PpIX maps, where the mean relative difference was $10.5 \%$ for $\mu_{\mathrm{a}}$ and $\mu_{\mathrm{s}}^{\prime}$ over the range of 0.2 to $5 \mathrm{~mm}^{-1}$, and there was no systematic difference apparent. Hence, there may be a degree of "self-correction" in the derived values for [PpIX]. Moreover, 

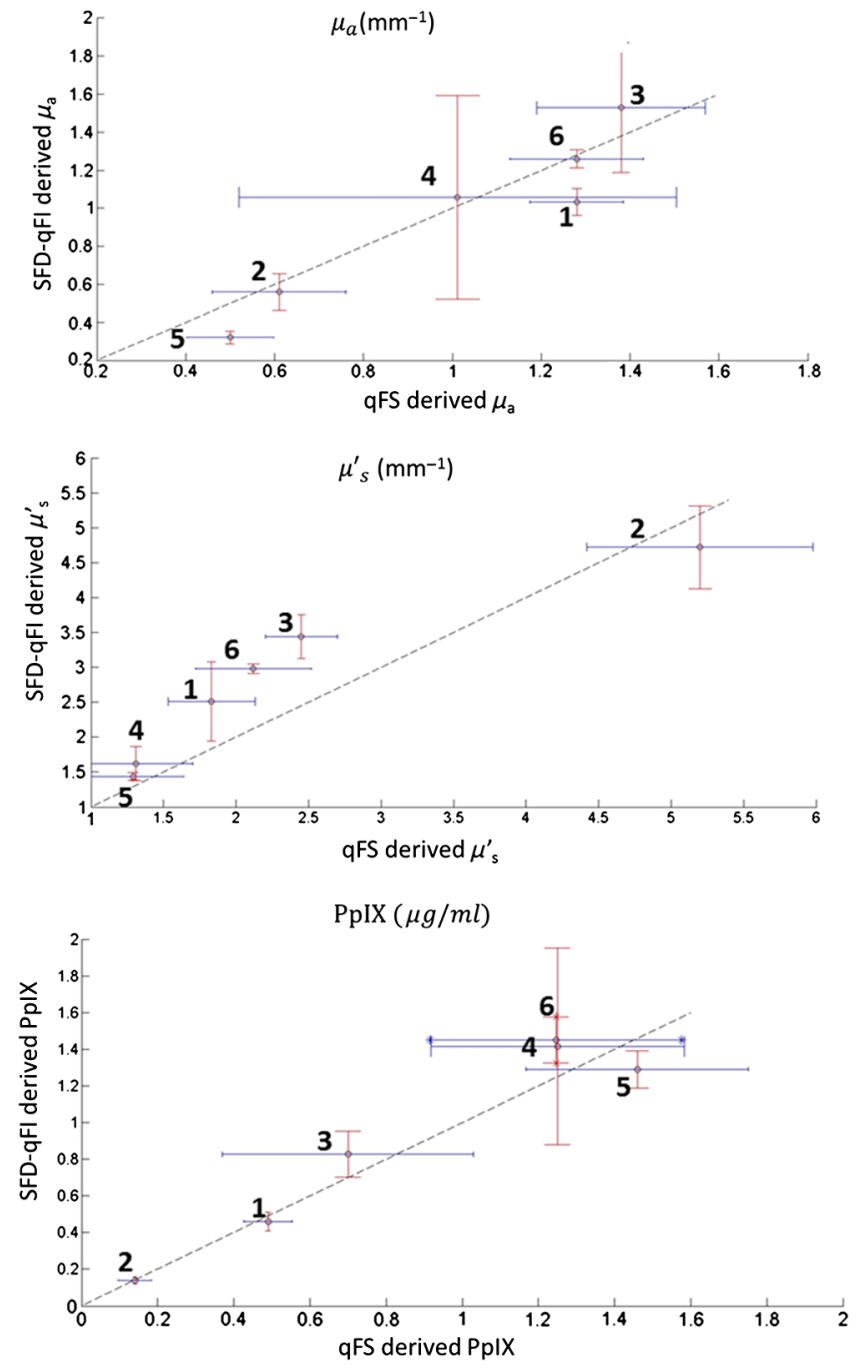

Fig. 6 Scatter plots of the mean values for $\mu_{\mathrm{a}}, \mu_{\mathrm{s}}^{\prime}$, and [PpIX] derived by SFD-qFI against the values obtained from qFS for in situ (rats \#1 to 3 ), ex vivo (rats \#4,5), and in vivo (rat \#6) measurements. The errors are the \pm 1 standard deviation within the tumor ROI. The lines of identity are also indicated (dashed lines).

statistical analysis showed that there is no significant difference in the values of $\mu_{\mathrm{a}}(p=0.586), \mu_{\mathrm{s}}^{\prime}(p=0.708)$, and [PpIX] ( $p=0.760)$ calculated by qFS and SFD-qFI at the $95 \%$ significance level. Finally, a third independent and well-established method for measuring [PpIX], based on fluorescence spectroscopy of ex vivo homogenized tissue, ${ }^{20}$ correlated well with both $\mathrm{qFS}$ and $\mathrm{qFI}(p>0.1)$.

\section{Discussion and Conclusions}

The goal of fluorescence guidance during (brain) tumor surgery is to maximize the completeness of tumor resection to limit recurrence and increase the efficacy of subsequent chemotherapeutic agents and radiation therapies. Because of the invasive and diffusive nature of gliomas as well as the need to preserve functional tissue, achieving optimal tumor resection is difficult using standard-of-care surgical approaches based on white light visualization combined with preoperative or intraoperative MRI. ${ }^{1-4}$ Qualitative ALA-PpIX fluorescence imaging during resection provides superior tumor-to-normal contrast, resulting in improved completeness of resection and survival outcomes for patients with high-grade glioma. ${ }^{1-4}$ However, it has not been sufficiently robust in the case of low-grade gliomas that generally have much lower PpIX concentrations, and even in high-grade disease, the subjective and qualitative interpretation of the fluorescence imaging has limited the resection to areas of "strong" fluorescence. ${ }^{1}$ These characteristics of qualitative ALA-PpIX fluorescence imaging are an impediment to consistent dissemination and clinical adoption of the technique. The high light attenuation of tissues, particularly for the violet/ blue wavelengths of PpIX fluorescence excitation, severely limits quantification of the PpIX concentration in vivo. ${ }^{6}$ Using an intraoperative point fluorescence/diffuse reflectance probe, quantitative measurements have been realized, demonstrating increased sensitivity and specificity in detecting residual tumor tissue at the end of white light resection as well as detecting nonvisible fluorescence from low-grade gliomas. ${ }^{6}$ However, such point-by-point sampling is time consuming (although each measurement is done in a fraction of a second), and undersampling may result in missing residual tumor tissue. Hence, there is interest in rapid, wide-field quantitative fluorescence imaging, especially if this can achieve comparable accuracy as the point measurement approach. This has been demonstrated here in a preclinical glioma model in rats using a full model-based approach enabled by SFDI.

Due to the infiltrative nature of the RG2 tumor model, a larger portion of the bulk tumor grew deeper $(>1.5 \mathrm{~mm})$ than initially intended, so full craniotomy and, in two cases, removal of superficial brain tissue (rats \#1, 2) was required. Removal of the dura to reduce specular reflection artifacts caused excessive bleeding, which overwhelmed the in vivo fluorescence and reflectance images (rat \#6). These problems necessitated modification of the experimental protocol, including the use of in situ/postmortem and ex vivo measurements (rats \#4, 5). However, these are limitations of the rat glioma model rather than of the method or instrument and will not apply in the clinic. Although the accuracy of the recovered tissue optical properties and [PpIX] using SFD-qFI was somewhat less than with the point-probe technique, the only systematic error was the slight overestimation of the scattering coefficient. The quantitative imaging also clearly revealed the intratumoral heterogeneities in both the tissue optical properties and the PpIX concentration.

The minimum [PpIX] value detected by SFD-qFI from the in situ rat brain images was $44 \mathrm{ng} / \mathrm{ml}$ in tumor and $13 \mathrm{ng} / \mathrm{ml}$ in normal brain. These should be compared with 11 and $8.6 \mathrm{ng} / \mathrm{ml}$ determined by qFS. The loss of sensitivity may be due in part to "spill over" or cross talk between image pixels and in part to sampling larger tissue volumes that introduces more tissuerelated distortion and attenuation of weak fluorescent signals. Nevertheless, compared with the raw fluorescence images, extracting the true [PpIX] values significantly improved the sensitivity, allowing detection of nonvisible fluorescent tumor, as seen by the example of rat \#2 (Fig. 2). Moreover, the quantitative PpIX maps showed enhanced tumor-to-normal contrast as seen by the example of rat \#1 and true tumor extent as seen in the example of rat \#3 (Fig. 2). It is important to note the differences in the sampled tissue volumes measured by each method as well as the differences in PpIX's fluorescence quantum efficiency. Both differences stem from the difference in the excitation wavelengths (405 versus $440 \mathrm{~nm}$ ) used for qFS compared with SFD-qFI. PpIX has its maximum absorption coefficient at $405 \mathrm{~nm}$, whereas the $440-\mathrm{nm}$ planar excitation will have a larger penetration depth $(0.45$ versus $0.25 \mathrm{~mm})$ and more 
excitation light is available to be absorbed by the tumor during wide-field imaging. As the three quantitative parameters were not seen to be significantly different when measured by the probe versus SFDI, the longer penetration depth during widefield imaging may have compensated for the suboptimal excitation wavelength.

This study also showed an added advantage of SFD-qFI over not only conventional qualitative FGR but also point qFS. The [PpIX] maps recapitulate the true PpIX distribution across the whole tumor, which is known to vary with ALA uptake and differences in the heme-biosynthesis pathway. A common observation in all rats was that the PpIX accumulation was significantly higher in regions with higher blood content, as seen by comparing the [PpIX] and $\mu_{a}$ maps in Fig. 4. Several other studies have shown a similar relationship between microvascular density and $[\mathrm{PpIX}] .{ }^{21-23}$ For example, Valdes et al. ${ }^{21}$ used surgically biopsied tissue to measure gadolinium (Gd) concentration (used in MRI) against [PpIX] in visible and nonvisible fluorescent tumor samples from nine patients with varying glioma grade. For each biopsy, a third sample was prepared for immunohistochemistry of microvascular density. ${ }^{21}$ Not only did the ex vivo Gd concentration correlate strongly with increased microvascular density, as expected, but also with the [PpIX] levels in both qualitative FGR-visible and nonvisible tumors, suggesting a common mechanism of vasculature leakiness on the accumulation of PpIX and Gd. In fact, [PpIX] better predicted neovascularization than did Gd. ${ }^{21}$ Samkoe et al. ${ }^{22}$ made similar observations in which ex vivo sections from an orthotopic preclinical glioma model had [PpIX] values that correlated with regions of $\mathrm{Gd}$-enhancement seen on in vivo T1-weighted MRI, but [PpIX] showed superior sensitivity to predicting tumor vascularity. Unmasking the fluorescent signal from highly vascularized tumors is critical as these tumors are more aggressive and tend to migrate beyond the primary site. ${ }^{21}$ It may be possible to use the total hemoglobin content and oxygen saturation of the tissue, which can be calculated from the tissue absorption spectra, as surrogate intrinsic biomarkers for microvascular density, and indeed these parameters derived in patients by the point-probe instrument have been shown to increase further the accuracy of residual glioma tissue detection. ${ }^{24}$

We are able to extract [PpIX] in vivo by decoupling the intrinsic fluorescence signal from biophysical and physiological distorting effects, based on recovering 2-D maps of $\mu_{\mathrm{a}}$ and $\mu_{\mathrm{s}}^{\prime}$. This is possible with SFDI, although it has limitations in tissue with high absorption. Our system was optimized to recover tissue optical properties in the subdiffusive regime down to a reduced transport albedo of $0.33 .{ }^{18}$ Despite implementing SFDI at higher spatial frequencies than typically used in the diffusion regime, $\mu_{\mathrm{a}}$ and $\mu_{\mathrm{s}}^{\prime}$ may not be as fully decoupled as they were in our previous phantom study ${ }^{18}$ that allowed sampling at spatial frequencies up to $1.7 \mathrm{~mm}^{-1}$. In that study, ${ }^{18}$ we had shown that higher spatial frequencies were required to accurately separate absorption from scattering. This is analogous to choosing shorter source-detector separations in the real space domain when tissue scattering is high, an optimization step implemented by the intraoperative probe. ${ }^{19}$ This was not possible in the small rat brain in which the highest spatial frequency that our current DMD array and imaging system can resolve is $1 \mathrm{~mm}^{-1}$ for a $3 \mathrm{~cm} \times 3 \mathrm{~cm}$ field of view. For future preclinical SFDI measurements in the subdiffusive regime, a higher resolution DMD array such as the 0.9 WQXGA Type A DMD series from Texas Instruments will be required. This should not be a significant issue in open-field surgery in patients, although it may be relevant in, e.g., laparoscopic surgery or endoscopy where the field of view is restricted. Work is in progress to implement the SFD-qFI system on a surgical microscope platform for further optimization and first clinical evaluations. ${ }^{25}$ Despite some systematic errors in deriving the optical properties by SFDI under our experimental conditions, [PpIX] can be retrieved with an accuracy approaching that of the point-probe $\mathrm{qFS}^{7}$ While we acknowledge that the rather long acquisition times applied here will interrupt the surgeon's work flow in the operating room, implementing SFDI at one spatial frequency, referred to as single snap shot imaging (SSOP), is now possible, ${ }^{26}$ where imaging and postprocessing of the modulated images can be recovered in 50 to $100 \mathrm{~ms}$. As for fluorescence imaging, replacing the CCD sensor with an EMCCD-based sensor allows PpIX detection down to $10 \mathrm{ng} / \mathrm{ml}$ with exposure times of 5 to $10 \mathrm{~ms}$. Both modifications, SSOP- and EMCCD-based detection, will facilitate translating qFI to the operating room. ${ }^{9}$

Finally, while the objective here was to enhance the accuracy of state-of-the-art PpIX FGR, which utilizes violet/blue light excitation, both qualitative PpIX FGR and SFD-qFI are limited to superficial tumors up to a depth of about $1 \mathrm{~mm}$ below the tissue surface. Our recent clinical implementation of PpIX FGR under red-light excitation ${ }^{27}$ has shown additional benefit by identifying deep-seated tumors located up to $5 \mathrm{~mm}$ below the surface. Hence, we are currently investigating the use of SFDI using 625-nm excitation light to interrogate subsurface PpIX-rich tumors and recovering both the depth and the PpIX concentration at that depth.

\section{Disclosures}

None of the authors have any financial interest to declare relating to the work in this paper.

\section{Acknowledgments}

This work was supported by the National Institutes of Health grant \# R01 NS052274. The authors would like to thank Carolyn Nui and Emily Chen in assisting with the animal preparations.

\section{References}

1. W. Stummer et al., "Extent of resection and survival in glioblastoma multiform: identification of and adjustment for bias," Neurosurgery 62, 564-576 (2008).

2. T. Hollon et al., "Advances in the surgical management of low-grade glioma," Semin. Radiat. Oncol. 25, 181-188 (2015).

3. D. W. Roberts et al., "Adjuncts for maximizing resection: 5-aminolevuinic acid," Neurosurgery 59, 75-78 (2012).

4. W. Stummer et al., "Fluorescence-guided surgery with 5-aminolevulinic acid for resection of malignant glioma: a randomized controlled multicenter phase III trial," Lancet Oncol. 7, 392-401 (2006).

5. L. Lilge et al., "The sensitivity of normal brain and intracranially implanted VX2 tumor to interstitial photodynamic therapy," $B r . J$. Cancer 73, 332-343 (1996).

6. P. A. Valdés et al., "Quantitative fluorescence in intracranial tumor: implications for ALA-induced PpIX as an intraoperative biomarker," J. Neurosurg. 115, 11-17 (2011).

7. A. Kim et al., "Quantification of in vivo fluorescence decoupled from the effects of tissue optical properties using fiber-optic spectroscopy measurements," J. Biomed. Opt. 15, 067006 (2010).

8. P. A. Valdés et al., "A spectrally constrained dual-band normalization technique for protoporphyrin IX quantification in fluorescence-guided surgery," Opt. Lett. 37, 1817-1819 (2012). 
9. M. Jermyn et al., "Improved sensitivity to fluorescence for cancer detection in wide-field image-guided neurosurgery," Biomed. Opt. Express 6, 5063-5074 (2015).

10. D. J. Cuccia et al., "Quantitation and mapping of tissue optical properties using modulated imaging," J. Biomed. Opt. 14, 024012 (2009).

11. B. S. Nichols et al., "A quantitative diffuse reflectance imaging (QDRI) system for comprehensive surveillance of the morphological landscape in breast tumor margins," PLoS One 10, e0127525 (2015).

12. J. T. Nguyen et al., "A novel pilot study using spatial frequency domain imaging to assess oxygenation of perforator flaps during reconstructive breast surgery," Ann. Plast. Surg. 71, 308-315 (2013).

13. A. M. Laughney et al., "System analysis of spatial frequency domain imaging for quantitative mapping of surgically resected breast tissues," J. Biomed. Opt. 18, 036012 (2013).

14. B. Yang, M. Sharma, and J. W. Tunnell, "Attenuation-corrected fluorescence extraction for image-guided surgery in spatial frequency domain," J. Biomed. Opt. 18, 080503 (2013).

15. S. D. Konecky et al., "Spatial frequency domain tomography of protoporphyrin IX fluorescence in preclinical glioma models," J. Biomed. Opt. 17, 056008 (2012).

16. R. B. Saager et al., "Quantitative fluorescence imaging of protoporphyrin IX through determination of tissue optical properties in the spatial frequency domain," J. Biomed. Opt. 16, 126013 (2011).

17. S. C. Kanick et al., "Sub-diffusive scattering parameter maps recovered using wide-field high-frequency structured light imaging," Biomed. Opt. Express 5, 3376-3390 (2014).

18. M. Sibai et al., "Quantitative spatial frequency fluorescence imaging in the sub-diffusive domain for image-guided glioma resection," Biomed. Opt. Express 6, 4923-4933 (2015).

19. A. Kim et al., "A fiberoptic reflectance probe with multiple sourcecollector separations to increase the dynamic range of derived tissue optical absorption and scattering coefficients," Opt. Express 18, 5580-5594 (2010)

20. L. Lilge, C. O'Carroll, and B. C. Wilson, "A solubilization technique for photosensitizer quantification in ex vivo tissue samples," J. Photochem. Photobiol. 39, 229-235 (1997).

21. P. A. Valdés et al., "Gadolinium and 5-aminolevulinic acid-induced protoporphyrin IX levels in human gliomas: an ex vivo quantitative study to correlate protoporphyrin IX levels and blood-brain barrier breakdown," J. Neuropathol. Exp. Neurol. 71, 806-813 (2012).

22. K. S. Samkoe et al., "Protoporphyrin IX fluorescence contrast in invasive glioblastomas is linearly correlated with Gd enhanced magnetic resonance image contrast but has higher diagnostic accuracy," J. Biomed. Opt. 16, 096008 (2011).

23. M. Olivo and B. C. Wilson, "Mapping ALA-induced PpIX fluorescence in normal brain and brain tumor using confocal fluorescence microscopy," Int. J. Oncol. 25, 37-45 (2004).

24. P. A. Valdés et al., "Combined fluorescence and reflectance spectroscopy for in vivo quantification of cancer biomarkers in low- and high-grade glioma surgery," J. Biomed. Opt. 16, 116007 (2011).

25. L. M. Angulo-Rodrigues et al., "An intraoperative spectroscopic imaging system for quantification of protoporphyrin IX during glioma surgery," Proc. SPIE 9690, 96900F (2016).

26. M. Van de Giessen et al., "Real-time imaging of tissue optical properties and surface profile using 3D-SSOP," Proc. SPIE 9319, 931910 (2015).

27. D. W. Roberts et al., "Red light excitation of protoporphyrin IX fluorescence for subsurface tumor detection," J. Neurosurg. (2017) (in press).

Biographies for the authors are not available. 